EDITORIAL

\title{
Is there any place for oral anti-restenotic treatment in the era of drug eluting stents?
}

\section{A H Gershlick}

Heart 2005;91:1377-1379. doi: 10.1136/hrt.2005.060954

Are drug eluting stents destined to become the standard of care for all patients undergoing percutaneous coronary intervention, or are alternative therapeutic approaches preferable under certain circumstances?

$\mathrm{P}$ ercutaneous coronary intervention (PCI) has become the dominant therapy for treating obstructive coronary disease. In Europe ratios of PCI to bypass graft surgery (CABG) vary from just over 2:1 in the UK to 6:1 in France and higher. Projected estimates suggest worldwide rates of PCI will increase to 3000 per million over the next five years. The reasons for the shift from coronary surgery towards PCI are multiple but include the introduction of stenting as the default procedure, improved stent design allowing interventionists to treat more complex, more distal lesions, the increase in operator skills, allowing for safer outcomes in higher risk procedures, as well as the introduction of adjunctive pharmacy, allowing for improved periprocedural results.

Important shortfalls in outcomes for multivessel stenting compared to multivessel surgery revolved around the difference in need for repeat intervention caused by restenosis for PCI patients, as reported in the ARTS 1, ERACI and SOS trials (excess revascularisation rates for PCI compared to CABG were $14 \%, 12 \%$, and $15 \%$, respectively). ${ }^{1-3}$ The importance of the development of drug eluting stents (DES) should therefore not be underestimated. Their history has been short, with early trials in simple lesions yielding clinical recurrence rates of less than 5\%, which have now been confirmed as reproducible in studies involving more complex lesions. Thus the E-SIRIUS ${ }^{4}$ and TAXUS IV $^{5}$ studies suggest that overall target lesion revascularisation rates of $3-4 \%$ can be anticipated with DES, and the e-Cypher registry of 15000 patients supports the biological efficacy of these devices in the real, complex world of chronic total occlusions, long lesions, in-stent restenosis, and the treatment of lesions in saphenous vein grafts. ${ }^{6}$

Because of DES, PCI appears at last able to match CABG in terms of medium term need for repeat procedures, with evidence of such coming from the ARTS 2 data, which were presented in late 2004. In this study overall DES major adverse cardiac events (MACE) at six months was $6.4 \%$ (target lesion revascularisation (TLR) $4.7 \%)$, compared to the previous ARTS I outcomes of 9\% MACE for CABG (TLR 3.3\%) and $20 \%$ (TLR 11.6\%) for ARTS 1 bare metal stent group. It seems reasonable to assume therefore that DES will become the standard of care for all patients undergoing PCI procedures in the foreseeable future. Therefore, are there any shadows on the DES horizon; any reasons to believe that alternative strategies will have a place or indeed may be needed or advantageous in any particular circumstances, as opposed to a future "DES only" world?

\section{ORAR STUDY}

This is the subtext question raised by the publication in this issue of Heart of the ORAR study by Rodriguez et al, ${ }^{7}$ who have assessed the impact of one month of oral rapamycin on outcome following bare metal stenting.

The ORAR study assessed the tolerability (measured by incidence of side effects) of different blood concentrations of systemically administered rapamycin, as well as their effects on standard angiographic outcomes measures at follow up. The study is small and observational. It is interesting that although the dose was the same in both phases of the study ( $2 \mathrm{mg} /$ day), they were able, through the addition of another frequently used cardiovascular drug (diltiazem $180 \mathrm{mg}$, which has been shown in renal transplant patients to increase blood concentration of rapamycin), to increase blood concentrations from mean $6.2 \mathrm{ng} / \mathrm{ml}$ to $9.3 \mathrm{ng} / \mathrm{ml}$. Various rather tortuous manipulations of the data (including receiver operating characteristic (ROC) curves) lead them to suggest that a concentration of $\geqslant 8 \mathrm{ng} / \mathrm{ml}$ was the "effective" concentration. Those patients in phase II of the study ( $>8 \mathrm{ng} / \mathrm{ml}$ ) had a target vessel revascularisation (TVR) rate of $12 \%$, albeit in a study with small numbers (that is, 5/42 patients needed a repeat procedure). While most DES studies report in-stent and in-lesion restenosis rates/late loss, this differentiation is perhaps less important in a study like ORAR where the effective agent is being given systemically when there should be no need to take account of the fact that there may be balloon injured regions without local drug cover.

\section{EFFECT OF ORAL RAPAMYCIN}

While the reported data from ORAR are limited in being derived from a small observational uncontrolled study, there are a number of

Abbreviations: $C A B G$, coronary artery bypass graft surgery; DES, drug eluting stents; MACE, major adverse cardiac events; $\mathrm{PCl}$, percutaneous coronary intervention; TLR, target lesion revascularisation; TVR, target vessel revascularisation 
messages that emerge. It is clear that that administration of diltiazem can indeed increase oral rapamycin blood concentrations. The drug appears relatively well tolerated $(31 \%$ side effects in phase II, but with only two patients needing to discontinue the drug) and that at blood concentrations of around $8 \mathrm{ng} / \mathrm{ml}$ or above, in-stent restenosis was only $6.2 \%$, with TVR rates of $12 \%$. There does appear to be an inverse discrepancy ("clinical" restenosis is usually lower than angiographic restenosis) and is likely to be caused by the combination of reporting TVR rather than TLR, together with this being reported at one year in a small number of subjects, as well as failure in the paper to correlate adequately the angiographic outcomes fully with the clinical outcomes.

The overall impression gained from this report is that oral rapamycin appears to have outcome benefits that are better than have been seen in bare metal stent studies, but perhaps not as good as those in DES trials. This is supported by the finding that late loss was $0.6 \mathrm{~mm}$ in the high blood concentration group (as opposed to the rather high $1.1 \mathrm{~mm}$ in the lower blood concentration group). This late loss of $0.6 \mathrm{~mm}$ is up to $0.2-0.3 \mathrm{~mm}$ higher than the best results for DES trials, but lower than the $0.9 \mathrm{~mm}$ or so expected in bare metal stent studies. The authors quite rightly point out, however, that we have little idea of the precise relation between late loss and outcome, as was shown in the ENDEAVOR 1 trial where a late loss of $0.58 \mathrm{~mm}$ was associated with a low clinical event rate. Currently, the relation between late loss and clinical outcome appears low and similar at any value less than $0.6 \mathrm{~mm}$ and clinical outcome only starts to increase exponentially at levels greater than $0.6 \mathrm{~mm}$.

Can there ever be a role for such agents and what studies would convince us of their true place and benefit. Taking the second question first, the ORAR trial is the latest in a number of small, observational non-randomised studies of this drug in patients undergoing coronary intervention. ${ }^{8}{ }^{9}$ It is therefore important to await the randomised placebo controlled trials (including ORAR II) but also anticipate that there will need to be others that compare oral rapamycin with Cypher/ rapamycin or even paclitaxel and other DES (which presumably would need to be large, non-inferiority studies).

Those observational studies that have been published confirm that this agent is associated with a late loss likely to be in the range of $0.6 \mathrm{~mm}$ and that binary restenosis and TLR is in the range of $6-8 \%$, although in some a higher incidence of intolerability was encountered. ${ }^{9}$ If, for the sake of argument, we assume that future appropriate studies indicate that use of oral rapamycin results in clinical outcomes that are better than bare metal stents alone but slightly worse than DES (say by 3-4 percentage points), and that tolerability and side effect profile are acceptable, the question to be asked is under what circumstances would we consider using such a strategy? In which patients would we prefer not to use DES? Cost of DES is clearly an issue, irrespective of arguments about cost effectiveness.

\section{RISK LOWERING STRATEGY}

Consideration of a risk lowering strategy, that is also low cost, may be particularly pertinent if, for example, multivessel ("surgically equivalent") revascularisation were being undertaken. One might imagine a strategy of one month oral rapamycin for multivessel stenting in lower risk circumstances (larger arteries, short lesions) where bare metal stents were being deployed. Such a strategy would have the advantage of further reducing restenosis risk at lower cost in such patients where each lesion carries an individual but potentially cumulative low risk. In developing countries where there is the potential for treating millions of patients percutaneously for coronary artery disease, the current cost of
DES is untenable. For such patients reducing the need to return for reintervention may make oral rapamycin an attractive therapeutic option. For multi-stent procedures the difference may be between protecting each lesion with an expensive DES or protecting all lesions with systemic rapamycin. It has to be effective and shown to be cost effective, however.

In certain higher risk patient subgroups (such as insulin dependant diabetics \pm small vessel stenting) oral rapamycin and a DES may help reduce the still higher than acceptable risk of restenosis that is currently reported with DES. Additionally bifurcation lesion event rates have not in the main been improved by DES. Technical difficulties in not only producing a true bifurcation stent, but even coating adequately those side branch access stents that are available, are well recognised problems. The currently favoured procedure is provisional stenting of the side branch with a high threshold for side branch stenting. A study assessing the event rates following bifurcation intervention (by whatever technique) following pre-intervention loading and postintervention administration of oral rapamycin would be a worth while exercise.

\section{LATE STENT THROMBOSIS}

Finally, the issue of late stent thrombosis with DES will not go away. ${ }^{10}$ It is still unclear whether it is real or not, but if it is then it is possibly due to the polymer or the delayed reendothelialisation consequent on longer term drug release. While long term (forever?) combined antiplatelet treatment may be the answer, this may reduce cost/efficacy and may not cover the times when general surgical procedures are needed, as is likely throughout a patient's antiplatelet dependant life. If oral rapamycin (or another effective antirestenotic agent) proved efficacious then re-endothelialisation time (and therefore antiplatelet need) could be reduced to the bare metal stent one month period.

While accepting the important impact that DES have had, we should continue to be critically aware of the problems associated with their use. It is important that studies such as that by Rodriguez et $\mathrm{al}^{7}$ are published, to keep the options open. For example, the IMPRESS study of oral steroid administration appears to show benefit (late loss $0.39 \mathrm{~mm}$ versus control $0.85 \mathrm{~mm}$ and restenosis in $7 \%$ of treated patients), ${ }^{11}$ but confirmation in appropriate studies appears limited by funding issues. It is important that robust studies of all such agents are now undertaken, to allow us to truly evaluate their benefit and tolerability and thereafter assess whether there is a role for them in interventional cardiology. It may not yet be time to assume DES are the panacea.

\section{REFERENCES}

1 Serruys PW, Unger F, Sousa JE, et al. Comparison of coronary-artery bypass surgery and stenting for the treatment of multivessel disease. N Eng J Med 2001;344:1117-24.

2 Rodriguez A, Bernardi V, Navia J, et al. Argentine randomized study: coronary angioplasty with stenting versus coronary bypass surgery in patients with multiplevessel disease (ERACI II): 30-day and one-year follow-up results. J Am Coll Cardiol 2001;37:51-8.

3 The SOS Investigators. Coronary artery bypass surgery versus percutaneous coronary intervention with stent implantation in patients with multivessel coronary artery disease (the stent or surgery trial): a randomised controlled trial. Lancet 2002;360:965-70.

4 Schofer J, Schluter M, Gershlick AH, et al for the E-SIRIUS Investigators. Sirolimus-eluting stents for treatment of patients with long atherosclerotic lesions in small coronary arteries: double blind, randomised controlled trial (ESIRIUS). Lancet 2003;362:1093-9.

5 Stone G, Ellis S, Cox D, et al. A polymer based paclitaxel eluting stent in patients with coronary artery disease. N Engl J Med 2004;350:221-31.

6 Urban P, Gershlick A, Guagliumi G, et al. MACE predictors after implantation of sirolimus-eluting stents: a report form the e-Cypher registry. Am J Cardiol 2004;94:68E

7 Rodriguez AE, Rodriguez Alemparte M, Vigo CF, et al. Role of oral rapamycin to prevent restenosis in patients with de novo lesions undergoing coronary 
stenting: results of the Argentina single centre study (ORAR trial). Heart 2005:91:1433-7.

8 Hausleiter J, Kastrati A, Mehilli J, et al for the OSIRIS Investigators. Randomized, double-blind, placebo-controlled trial of oral sirolimus for restenosis prevention in patients with in-stent restenosis: the oral sirolimus to inhibit recurrent in-stent stenosis (OSIRIS) trial. Circulation 2004;110:790-5.

9 Waksman $R$, Ajani $A E$, Pichard $A D$, et al. Oral rapamycin to inhibit restenosis after stenting of de novo coronary lesions: the ora rapamune to inhibit restenosis (ORBIT) study. J Am Coll Cardiol 2004:44:1386-92.

10 McFadden EP, Stabile E, Regar E, et al. Late thrombosis in drug-eluting coronary stents after discontinuation of antiplatelet therapy. Lancet 2004:364:1519-21.

11 Versaci F, Gaspardone A, Tomai F. Immunosuppressive therapy for the prevention of restenosis after coronary artery stent implantation (IMPRESS study). J Am Coll Cardiol 2002;40:1935-42.

\section{IMAGES IN CARDIOLOGY}

doi: $10.1136 / \mathrm{hrt} .2005 .063867$

\section{An intrapericardial pheochromocytoma presented with paroxysmal attacks of hypertension and palpitation: typical presentation with unusual location of the fumour}

A 60 year old man came to the emergency room because of severe chest pain, headache, and palpitations accompanied by facial flushing and sweating. On admission his blood pressure was $200 / 100 \mathrm{~mm} \mathrm{Hg}$, and pulse rate 110 beats/min. ECG showed no specific findings for myocardial ischaemia. A computed tomography scan to rule out aortic dissection showed, however, a $8 \times 5 \mathrm{~cm}$ sized dumbbell shaped mass within the pericardial space between the aorta and the main pulmonary artery. The mass abuts the ascending aorta, main pulmonary artery, and left main and proximal left anterior descending artery; however, invasion or adhesion was excluded by demonstrating sliding motion between the mass and the adjacent structures with cine magnetic resonance imaging (MRI) (panels A, B). A 24 hour urine study revealed raised concentrations of noradrenaline (norepinephrine) (233.7 $\mu \mathrm{g} /$ day, normal range $15-80 \mu \mathrm{g} /$ day) and vanillynmandelic acid $(23.29 \mathrm{mg} /$ day, normal range $<8 \mathrm{mg}$ /day). Abdominal MRI showed, however, no adrenal mass. A $\left[{ }^{131} \mathrm{I}\right]$ metaiodobenzyl-guanidine scintiscan revealed a hot uptake spot anatomically corresponding to the intrapericardial mass (panel C). The dumbbell shaped mass between the aorta and the main pulmonary artery was successfully excised (panel D) under cardiopulmonary bypass without damaging adjacent vessels after treatment with phenoxybenzamine and propranolol for four weeks.

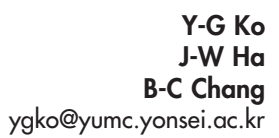

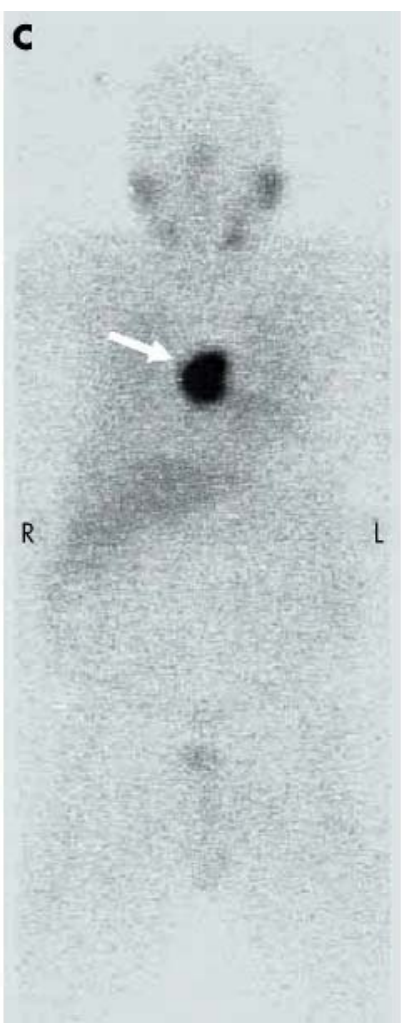

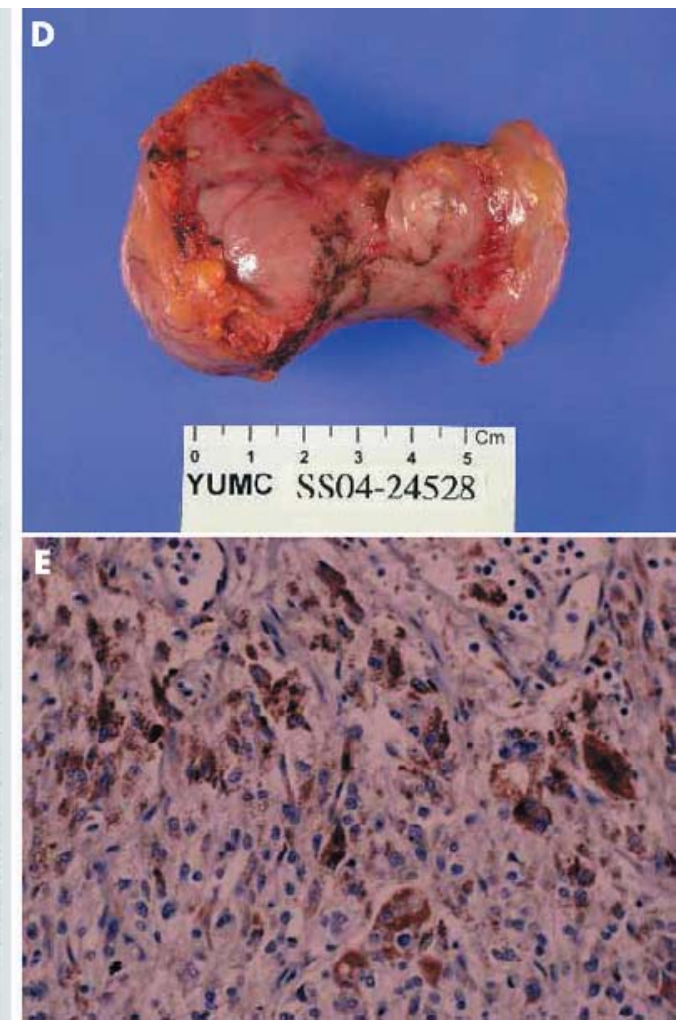

Intrapericardial pheochromocytoma. Panels A, B: cardiac magnetic resonance image of dumbbell shaped mass (asterisk) between the aorta and the main pulmonary artery. Panel C: hot uptake spot (white arrow) in [ $\left.{ }^{131} \mathrm{I}\right]$ metaiodobenzyl-guanidine scintiscan. Panel D: gross appearance. Panel E: histologic appearance of the tumour strongly immunoreactive for synaptophysin and chromogranin. Ao, aorta; MPA, main pulmonary artery. 